\title{
LA AYUDA AL COMERCIO Y SU RELACIÓN CON LA COMPETITIVIDAD INTERNACIONAL DE LOS PAISES MENOS ADELANTADOS
}

\author{
AID FOR TRADE AND LEAST-DEVELOPED COUNTRIES' RELATIONSHIP WITH \\ INTERNATIONAL COMPETITIVENESS
}

A AJUDA AO COMÉRCIO E SUA RELAÇÃO COM A COMPETITIVIDADE INTERNACIONAL DOS PAÍSES MENOS DESENVOLVIDOS

\section{Kendall Ariana López Peña ${ }^{1}$ Raúl Gerardo Fonseca Hernández²}

\begin{abstract}
Resumen
La Organización Mundial del Comercio (OMC) posee un programa de cooperación no reembolsable dirigida a otorgar ayuda al comercio de los denominados países menos adelantados (PMA), los cuales se caracterizan por presentar un bajo desarrollo humano, reflejan problemas importantes en áreas estratégicas tales como infraestructura, procedimientos, instituciones y políticas de apoyo al comercio. El objetivo del programa es apoyar en la creación de capacidad comercial para promover la competitividad internacional de los PMA.

La investigación busca establecer la relación entre la Ayuda para el Comercio, otorgada por la OMC y su efecto en la Competitividad
\end{abstract}

Doi: http://dx.doi.org/10.15359/eys.23-54.1

Fecha de recepción: 20-03-2018. Fechas de reenvíos: 20-03-2018, 04-05-2018, 04-06-2018. Aceptado el 15-06-2018. Publicado el 01-07-2018.

${ }^{1}$ Máster en Relaciones Internacionales y Diplomacia. Académica e investigadora de la Escuela de Relaciones Internacionales de la Universidad Nacional, Heredia, Costa Rica. Correo electrónico: kendall.lopez.pena@una.ac.cr

${ }^{2}$ Máster en Economía del Desarrollo. Académico e investigador de la Escuela de Relaciones Internacionales de la Universidad Nacional; Heredia, Costa Rica y de la Universidad de Costa Rica, Sede Occidente, Costa Rica. Correo electrónico: raul.fonseca.hernandez@una.ac.cr

Kendall Ariana López Peña y Raúl Gerardo Fonseca Hernández

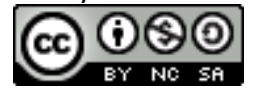

Revista Economía y Sociedad by Universidad Nacional is licensed under a CreativeCommons Reconocimiento-NoComercial- 
Internacional de los países sujetos a su apoyo (treinta y seis PMA, miembros de la OMC). Para lo cual se genera un índice de competitividad internacional conformado por las siguientes variables: competitividad interna, desarrollo humano, eficiencia de las exportaciones y apertura comercial.

El artículo concluye que existe una correlación débil entre la Ayuda para el Comercio, otorgada por la OMC y la Competitividad Internacional de los PMA. La efectividad de la ayuda podría mejorar si los PMA invierten en suministros de electricidad e infraestructura portuaria y aeroportuaria.

Palabras claves: ayuda para el Comercio; competitividad internacional; OMC; países menos adelantados

\begin{abstract}
The World Trade Organization's (WTO) non-reimbursable cooperation program aims to provide aid for trade to least-developed countries (LDCs). These countries are characterized by having a low Human Development Index (HDI) and major problems in core strategic areas such as infrastructure, procedures, institutions, and supportive trade policies. The program's objective is to develop trade capacity to enhance LDCs' international competitiveness.
\end{abstract}

The study aims to establish the relationship between Aid for Trade and its effect on the international competitiveness of the 36 WTO least-developed member countries. To draw this relationship, an international competitiveness index was developed, including variables such as domestic competitiveness, human development, exportation performance, and trade openness.

The article concluded that there is a weak relationship between Aid for Trade and its effect on LDCs' international competitiveness, which could improve if LDCs invest in electricity provisions together with port and airport infrastructure.

Keywords: Aid for Trade; international competiveness; WTO; leastdeveloped countries

2

Kendall Ariana López Peña y Raúl Gerardo Fonseca Hernández

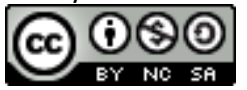

Revista Economía y Sociedad by Universidad Nacional is licensed under a CreativeCommons Reconocimiento-NoComercial- 


\section{Resumo}

A Organização Mundial do Comércio (OMC) possui um programa de cooperação não reembolsável dirigida a outorgar ajuda ao comércio dos denominados países menos desenvolvidos (PMD), caracterizados por apresentar um baixo desenvolvimento humano e refletir problemas importantes em áreas estratégicas, tais como infraestrutura, procedimentos, instituições e políticas de apoio ao comércio. O objetivo do programa é apoiar a criação de capacidade comercial para promover a competitividade internacional dos PMD.

A pesquisa busca estabelecer a relação entre a ajuda ao comércio, outorgada pela OMC e seu efeito na competitividade Internacional dos países sujeitos a seu apoio (trinta e seis PMD, membros da OMC). Para isso é gerado um índice de competitividade internacional conformado pelas seguintes variáveis: competitividade interna, desenvolvimento humano, eficiência das exportações e abertura comercial.

O artigo conclui que existe uma correlação débil entre a ajuda ao comércio, outorgada pela OMC, e a Competitividade Internacional dos PMD. A eficácia da ajuda poderia melhorar se os PMD investissem em fornecimento de eletricidade e infraestrutura portuária e aeroportuária.

Palavras-chave: ajuda ao comércio; competitividade internacional; OMC; países menos desenvolvidos

\section{Introducción}

Desde la Segunda Guerra Mundial, la mayoría de los países del mundo se han insertado en un sistema global multilateral, liderado por la Organización Mundial del Comercio (OMC), la cual busca reducir las barreras al comercio mundial. En ese sentido, los países han apostado al comercio internacional como un mecanismo potencial para alcanzar no solo el crecimiento económico, sino también el desarrollo y la competitividad internacional de cada uno.

Sin embargo, un importante conglomerado de países en desarrollo y en particular, los países menos adelantados (PMA) ${ }^{3}$ presentan claras dificultades para insertarse en este sistema multilateral y lograr maximizar los beneficios del comercio como herramienta para el desarrollo.

\footnotetext{
${ }^{3}$ Los países menos adelantados (PMA) (LDC por sus siglas en inglés) son un grupo de 49 países que se caracterizan por un reducido nivel de PIB per cápita, la falta de recursos humanos y un elevado grado de vulnerabilidad económica (Conferencia de las Naciones Unidas para el Comercio y Desarrollo [UNCTAD], 2015).
}

Kendall Ariana López Peña y Raúl Gerardo Fonseca Hernández

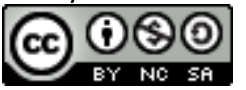

Revista Economía y Sociedad by Universidad Nacional is licensed under a CreativeCommons Reconocimiento-NoComercial- 
Estos territorios cuentan con limitadas capacidades en materia de infraestructura, procedimientos, instituciones y políticas para insertarse y competir en los mercados globales.

El principio de la OMC sobre el "Trato especial y diferenciado", establece una serie de flexibilidades y privilegios especiales en favor de los países en desarrollo. Dicho principio constituye la base de un conjunto de acciones, orientadas a dinamizar el comercio internacional de aquellos miembros menos adelantados. En este sentido, la OMC ha creado una institucionalidad y un diseño normativo que se ha materializado en el programa de cooperación no reembolsable denominado Ayuda para el Comercio de los PMA.

La presente investigación pretende analizar la existencia de una relación entre la ayuda al comercio y su efecto, si es que la hay, en la Competitividad Internacional de los PMA. Para lo cual se construye de un Índice de Competitividad Internacional (ICI), el cual está compuesto por las siguientes variables: competitividad interna, desarrollo humano, eficiencia de las exportaciones y apertura comercial.

El artículo pretende dar respuesta a las siguientes interrogantes: ¿cuál es la relación entre la ayuda al comercio y la competitividad internacional?, así como, ¿cuáles son las áreas qué debería enfocarse la ayuda al comercio de los PMA para mejorar la competitividad internacional de éstos? El documento se estructura en tres secciones. La primera sección, se analizan aspectos teóricos de la Ayuda al Comercio. En la segunda sección, se presenta la metodología propuesta para la elaboración del Índice de Competitividad internacional. En el tercer apartado, se divide en tres subsecciones, en las cuales se analizan los resultados referentes a los principales receptores y oferentes de la Ayuda al Comercio, el Índice de Competitividad internacional; y la relación entre la Ayuda al Comercio y la Competitividad Internacional. Finalmente, se exponen las principales conclusiones de la investigación.

\section{Marco referencial}

El marco referencial se divide en tres secciones para facilitar el análisis de los temas medulares que sustentan el análisis de los principales resultados de la investigación: en un primer apartado se muestran las principales teorías de las relaciones internacionales que explican la cooperación internacional desde una visión transnacionalista, institucionalizada en el marco de un régimen internacional; la segunda sección presenta a la OMC y al programa de Ayuda al Comercio de los PMA, en cuanto a su concepción y evolución en el tiempo; por último, se ofrecen referencias conceptuales y analíticas sobre la competitividad internacional.

4

Kendall Ariana López Peña y Raúl Gerardo Fonseca Hernández

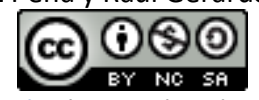

Revista Economía y Sociedad by Universidad Nacional is licensed under a CreativeCommons Reconocimiento-NoComercial- 


\section{Teorías de las Relaciones Internacionales sobre cooperación para el desarrollo}

En la disciplina de las Relaciones Internacionales existen distintas teorías y proposiciones que han intentado explicar los motivos de la cooperación entre Estados y el papel de las organizaciones internacionales en el Sistema Internacional. Destacan el grosiano, trasnacionalismo, el neoliberalismo, el institucionalismo, y más recientemente las posturas de los regímenes internacionales. Dichos enfoque generan un tratamiento teórico y conceptual que aportan un punto de vista singular, que sustenta el análisis que se realiza en la presente investigación.

Por un lado, el enfoque grosiano, plantea como la Cooperación Internacional debe garantizar la creación de instituciones y normas que limiten el conflicto y potencien los intereses mutuos. El enfoque clásico grosiano, analiza el papel de la cooperación internacional en el actual sistema internacional, el cual está constituido por: "un conjunto de actores, cuyas relaciones generan una configuración de poder (estructura) dentro de la cual se produce una red de interacciones (procesos) de acuerdo a determinadas reglas" (Barbé, 2007, p. 23).

Desde este enfoque se estipula como unidad de análisis la pluralidad de actores, que poseen problemáticas distintas, tales como medio ambiente, derechos humanos, seguridad alimentaria, ayuda al comercio; entre otros temas de interés de las agendas políticas de los países. Es por ello, que resulta ser una perspectiva más adecuada para explicar el papel de la cooperación internacional actual.

Desde la visión aportada por la teoría transnacionalista, más adelante profundizada por la teoría neoliberal, se valida la presencia de instituciones internacionales que tienen la capacidad de promover la cooperación en áreas en las que difícilmente se podría conseguir por parte de las naciones necesitadas. En este sentido, las agencias de cooperación estatales, instituciones u organismos no gubernamentales pueden especializarse en el otorgamiento y gestión de cooperación técnica o financiera, ya sea reembolsable o no, de tópicos particulares muy especializados. Un ejemplo de una institución transnacionalista que ofrece este tipo de cooperación es la OMC cuya ayuda se basa en temas comerciales a los países miembros.

La teoría institucionalista ha intentado explicar cómo la cooperación puede perdurar sin una formalización o legalización particular. Autores como Abbott y Snidal (citado por Goldstein, Kahler, Keohane y Slaughter, 2000), así como Keohane y Nye (1988), extienden el planteamiento institucionalista al explicar la legalización y las distintas variaciones que presenta. De modo tal, que para responder a una de las aristas de esta investigación y finalmente describir las operatividad de la OMC, es necesario no solo adoptar la conceptualización de Krasner (1983)

Kendall Ariana López Peña y Raúl Gerardo Fonseca Hernández

(c) (i) (8)

Revista Economía y Sociedad by Universidad Nacional is licensed under a CreativeCommons Reconocimiento-NoComercial- 
sobre regímenes internacionales ${ }^{4}$ sino también incorporar la definición de legalización de Goldstein, et al., (2000), que contiene tres criterios: "el grado en que las reglas son obligatorias, la precisión de esas reglas y la delegación de algunas funciones de interpretación, monitoreo e implementación a un tercero" (p. 387). Es así que, desde el concepto de Abbot, Keohane, Moravcsik, Slaughter y Snidal "una institución plenamente legalizada es una institución con altos niveles de obligación, precisión y delegación" (citado por Goldstein, et al., 2000, p. 396).

En este sentido, la institucionalización de la cooperación internacional genera un conjunto de normas, reglas y procedimientos para la toma de decisiones que configuran las expectativas, los intereses y el comportamiento de los actores en muchas dimensiones y difieren, entre ellas, según el alcance de sus reglas, los recursos disponibles para las organizaciones formales y su grado de diferenciación burocrática. En este sentido Goldstein, et al., (2000) indican que:

Una mayor institucionalización implica que las reglas institucionales gobiernan más el comportamiento de actores importantes más en el sentido de que un comportamiento que anteriormente estaba fuera del alcance de reglas particulares está ahora dentro de ese ámbito o que el comportamiento que estaba regulado anteriormente está ahora más profundamente regulado. (p. 387)

\section{La OMC y el programa de "ayuda al comercio" de los PMA}

La OMC es una organización creada para liberalizar el comercio, un foro de negociación donde acuden los gobiernos a resolver sus problemas comerciales. Así mismo, constituye un régimen

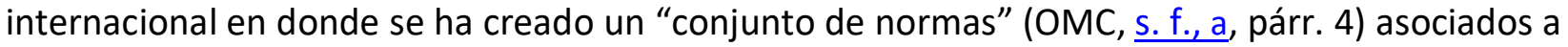
temas comerciales. Su núcleo está constituido por los acuerdos de la OMC, negociados y firmados por la mayoría de los países que participan en el comercio internacional. Estos documentos establecen las normas jurídicas fundamentales del comercio internacional. Los acuerdos son, esencialmente, contratos que obligan a los países a mantener políticas comerciales dentro de los límites convenidos.

El régimen internacional de la $\mathrm{OMC}$ presenta al mismo tiempo una serie de normativas, reglas y procedimientos entorno al área temática de "Ayuda para el Comercio". El objetivo principal de la "iniciativa de la ayuda para el comercio" es apoyar a los países en desarrollo, en particular a los PMA, a crear la capacidad comercial y la infraestructura que necesitan para beneficiarse de la apertura del comercio.

4 "Conjuntos de principios, normas, reglas y procedimientos de toma de decisiones implícitos o explícitos, alrededor de los cuales convergen las expectativas de los actores en área dada de las relaciones internacionales" (Krasner, 1983: 186).

6

Kendall Ariana López Peña y Raúl Gerardo Fonseca Hernández

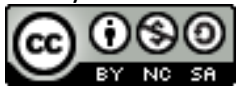

Revista Economía y Sociedad by Universidad Nacional is licensed under a CreativeCommons Reconocimiento-NoComercialCompartirlgual 4.0 Internacional License.

Creado a partir de la obra en http://www.revistas.una.ac.cr/index.php/economia 
El programa de ayuda al comercio tiende a institucionalizarse en el tiempo. En vista de que si bien la OMC no se constituye como un organismo meramente de financiación y su función principal sigue recayendo en el Sistema Multilateral de Comercio, las acciones de cooperación internacional dirigidas a países en desarrollo y en particular a los PMA, presentan una evolución en cuanto a las responsabilidades y actividades para obtener beneficios comerciales mayores y efectivos, en materia de oportunidades de acceso a los mercados.

El apoyo a los PMA nace en 2005 y ha evolucionado en el tiempo. Surge concretamente en la Conferencia Ministerial del 2005 celebrada en Hong Kong, en la cual se afirmó que la Ayuda para el Comercio debe de tener como finalidad la ayuda a los países en desarrollo. En dicha conferencia se afirma que:

La Ayuda para el Comercio deberá tener el objetivo de ayudar a los países en desarrollo, en particular a los PMA, a crear la capacidad de oferta y la infraestructura relacionada con el comercio que necesitan para poder aplicar los Acuerdos de la OMC y beneficiarse de ellos y, más en general, para aumentar su comercio. (OMC, s. f., b, p. 57)

En el 2006, el Equipo de Trabajo sobre la Ayuda para el Comercio de la OMC, señala que la Ayuda para el Comercio tiene por objeto "prestar asistencia a los países en desarrollo para que aumenten las exportaciones de bienes y servicios, se integren en el Sistema Multilateral de Comercio y se beneficien de la liberalización del comercio y el incremento del acceso a los mercados" (WTO, 2006, p. 1). El Equipo de Trabajo propone clasificar la Ayuda para el Comercio en seis categorías:

a) "Políticas y reglamentos comerciales, con inclusión de: formación de funcionarios de servicios comerciales, análisis de propuestas y posiciones y de su repercusión, apoyo a los colectivos nacionales interesadas para articular los intereses comerciales e identificar las ventajas e inconvenientes, cuestiones relativas a diferencias, apoyo institucional y técnico para facilitar la aplicación de acuerdos comerciales y adaptar y cumplir las normas.

b) Fomento del comercio, con inclusión de: promoción de las inversiones, análisis y apoyo institucional del comercio de servicios, servicios e instituciones de ayuda a las empresas, creación de redes de los sectores público y privado, comercio electrónico, financiación del comercio, promoción del comercio, análisis y desarrollo del mercado. c. Infraestructura relacionada con el comercio, con inclusión de obras de infraestructura.

c) Creación de capacidad productiva. 
d) Ajuste relacionado con el comercio, con inclusión de ayuda a los países en desarrollo para aplicar medidas complementarias que les permitan beneficiarse de la liberalización del comercio.

e) Otras necesidades relacionadas con el comercio. (citado en World Trade Organization, $\underline{2006}$, p. 2)

El apoyo ha evolucionado hasta tal punto, que en la Novena Conferencia Ministerial celebrada en Bali (Indonesia) en diciembre de 2013, los ministros de comercio exterior de los países integrados a la OMC muestran su satisfacción por los resultados logrados en el Examen Global de Ayuda para el Comercio, aplicado en julio de 2013. En dicha conferencia se adoptó un paquete que incluía la facilitación al comercio para productos agrícolas, tal como el algodón, el cual es de producción común entre los PMA, también se agrega la asistencia tanto técnica como administrativa, con el objetivo de poder crear mayor capacidad de comercio.

Entre el 2014 y el 2017 se profundiza el apoyo a los PMA por parte de la OMC. En este sentido se establecen los Programa de trabajo sobre Ayuda al Comercio (2014-2015) y (2016-2017), los cuales contienen una importante cantidad de acciones dirigidas al apoyo de los PMA. Por ejemplo, en el Programa de Trabajo 2016-2017 (citado en OMC, 2016) propone:

Analizar más detenidamente las limitaciones de la capacidad de oferta y de la infraestructura relacionada con el comercio a las que se enfrentan los países en desarrollo, prestando especial atención al comercio de servicios y la mejora de la infraestructura, $y$, por medio de estas actividades, contribuir a una mayor coherencia. (p. 1)

\section{La competitividad internacional}

Para lograr que los PMA aprovechen de mejor forma la ayuda al comercio, deben invertirse los recursos en áreas estratégicas que mejoren su competitividad internacional. La teoría económica muestra una serie de evoluciones en el concepto de competitividad internacional; iniciando con el planteamiento de la ventaja competitiva de las naciones de Michael Porter (Porter, 2007). En esta misma línea, Moon, Rugman y Verbeke (1995) (citado por C. Castellanos, J. Castellanos, Machado, Vila y Barbosa, 2012) explican que la base del éxito nacional se encuentra en la capacidad de las empresas para seguir creando valor a pesar de la competitividad internacional. Cho (1994) (citado por C. Castellanos, J. Castellanos, Machado, Vila y Barbosa, 2012) por su parte, propone que el éxito depende de la actuación de países y regiones similares, de que se consiga estar en una posición competitiva comparativamente superior y que perdure en el largo plazo.

Por su parte, los modelos del World Economic Forum (WEF) y del International Institute for Management Development explican que el éxito consiste en crear una serie de condiciones

8

Kendall Ariana López Peña y Raúl Gerardo Fonseca Hernández

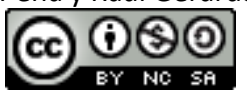

Revista Economía y Sociedad by Universidad Nacional is licensed under a CreativeCommons Reconocimiento-NoComercialCompartirlgual 4.0 Internacional License.

Creado a partir de la obra en http://www.revistas.una.ac.cr/index.php/economia 
microeconómicas y un entorno en el que las empresas puedan competir con éxito. De manera tal, que en la teoría económica tradicional la riqueza de las naciones se basaba en la dotación de factores, mientras en la teoría económica moderna son las elecciones estratégicas, las que conforman el entorno competitivo de una nación.

El Programa de Trabajo de la OMC sobre la Ayuda para el Comercio (citado en OMC, 2007) establece la creación de capacidad comercial como una condición necesaria para aumentar la competitividad internacional y mejorar los resultados comerciales de los países en desarrollo, en particular los PMA. Asimismo, en el Programa de Trabajo de la OMC sobre la Ayuda para el Comercio, se destaca que hay tres elementos clave que determinan la competitividad internacional y los resultados comerciales de un país: un buen marco normativo, buenas políticas y prácticas de facilitación del comercio y una buena base de capacidad comercial, en particular infraestructura relacionada con el comercio.

\section{Metodología}

Para el establecimiento de la relación entre la ayuda al comercio y su efecto en la Competitividad Internacional de los PMA se realiza un Índice de Competitividad Internacional (ICI), y se calcula la respectiva correlación entre ambas variables. Se toma como base el trabajo metodológico realizado por Fonseca (2012).

En la selección de variables que componen el índice se analiza distintas posturas teóricas sobre competitividad (Cho 1994, Moon, Rugman y Verbeke 1995; citado citado por C. Castellanos, J. Castellanos, Machado, Vila y Barbosa, 2012; Porter 2007) y se revisa diversos reportes que examinan la competitividad internacional de países tales como: Banco Mundial (s. f.); Programa de Trabajo de la OMC sobre la Ayuda al Comercio (2007), The Global Competitiveness Report 2015-2016 (WEF, s. f.) y IMD World Competitiveness Center (2014); OECD \& WTO (2015); Banco Mundial (2016). Al respecto López (2017) señala:

La disponibilidad de datos, de los treinta y seis PMA en un momento del tiempo determinado, fue un elemento necesario para determinar la selección de las variables y sub variables en estudio. Las variables seleccionadas fueron sometidas a una consulta de diez expertos instituciones gubernamentales nacionales e internacionales, empresa y organismos internacionales, aplicada entre los meses de setiembre y octubre de 2016, para validar su selección y determinar el peso relativo que tendrán en el índice. Los datos al tener diferentes unidades de medida (millones de USD, tiempo de exportación, porcentajes, etc.) deben estandarizarse con el propósito de que todos los datos sean comparables. (López, 2017, p. 34)

Kendall Ariana López Peña y Raúl Gerardo Fonseca Hernández

(c) (i) (8)

Revista Economía y Sociedad by Universidad Nacional is licensed under a CreativeCommons Reconocimiento-NoComercialCompartirlgual 4.0 Internacional License.

Creado a partir de la obra en http://www.revistas.una.ac.cr/index.php/economia 
El Índice de Competitividad Internacional explica la competitividad internacional por medio de cuatro subvariables y diez indicadores; distribuidos de la siguiente forma: cinco indicadores explican la competitividad interna; mientras dos exponen la eficiencia de las exportaciones y tres conforman la subvariable de apertura comercial. La Tabla 1 muestra la variable, subvariable, indicador y peso relativo según la encuesta aplicada.

Tabla 1

Sub variables, indicadores e importancia relativa

\begin{tabular}{|c|c|c|c|}
\hline Variable & Subvariable & Indicador & Peso \% \\
\hline Competitividad & Competitividad interna & Acceso a las cargas & $5,4 \%$ \\
\hline \multirow[t]{8}{*}{ Internacional } & & Suministros de electricidad & $5.4 \%$ \\
\hline & & Carreteras & $5.4 \%$ \\
\hline & & Infraestructura portuaria & $5.4 \%$ \\
\hline & & Infraestructura de transporte aéreo. & $5.4 \%$ \\
\hline & Eficiencia de las & Tiempo de exportaciones & $13 \%$ \\
\hline & exportaciones & Costo de las exportaciones & $13 \%$ \\
\hline & Apertura comercial $^{5}$ & Exportaciones + importaciones/PIB & $24 \%$ \\
\hline & Desarrollo Humano & Índice de Desarrollo Humano & $23 \%$ \\
\hline
\end{tabular}

Fuente: Elaboración propia; a partir de los resultados de la encuesta electrónica y de estadísticas del Central Intelligence Agency (2014c) y el OECD \& WTO (2015).

Los resultados del índice se clasifican en quintiles según su competitividad: alta competitividad, AC (entre 80 y 100), muy competitivo MC (entre 60 y 79), competitividad media (entre 40 y 59), poca competitividad PC (entre 20 y 39) y baja competitividad BC (entre 0 y 19).

La relación se muestra por medio de las siguientes ecuaciones.

Ecuación 1: Relación entre la variable dependiente de la Ayuda para el Comercio y la independiente de la Competitividad Internacional.

$$
\mathrm{Ac} \rightarrow \int\{\mathrm{Ci} ; \mathrm{EE} ; \mathrm{AC} ; \mathrm{IDH}\}
$$

Donde

Ac La Ayuda para el Comercio

Ci Competitividad Interna, la cual está en función ( $\int$ ) de la ecuación 2

\footnotetext{
${ }^{5}$ Para la construcción de la apertura comercial fue necesaria la recolección de estadísticas de las exportaciones, importaciones y PIB de cada uno de los 36 PMA.
}

10

Kendall Ariana López Peña y Raúl Gerardo Fonseca Hernández

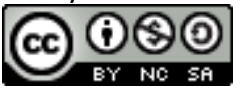

Revista Economía y Sociedad by Universidad Nacional is licensed under a CreativeCommons Reconocimiento-NoComercial- 
Ecuación 2: Competitividad interna

$$
C i \rightarrow \int\{A c ; S e ; C ; I p ; I a\}
$$

Donde:

Ac Acceso a las cargas

Se Suministro de electricidad

C Carreteras

Ip Infraestructura portuaria

la Infraestructura aeroportuaria

EE Eficiencia de las exportaciones, se expresa en la ecuación 3

Ecuación 3: Eficiencia de las exportaciones

$$
\mathrm{EE} \rightarrow \int\{\mathrm{Ce} ; \mathrm{Te}\}
$$

Donde:

Te Tiempo de exportaciones

$\mathrm{Ce} \quad$ Costo de las exportaciones

AC Apertura Comercial, la cual se enuncia en la ecuación 4

Ecuación 4: Apertura Comercial

$$
\mathrm{AC}=\frac{\mathrm{E}+\mathrm{M}}{\mathrm{PIB}}
$$

Donde:

E Exportaciones

M Importaciones

PIB Producto Interno Bruto

IDH Índice de Desarrollo Humano

El análisis de correlación se desarrolla a través del Coeficiente de Pearson, el cual devuelve el coeficiente de correlación producto o momento $r$ de Pearson, un índice adimensional acotado entre $-1,0$ y 1,0, ambos incluidos, que refleja el grado de dependencia lineal entre dos conjuntos de datos (Fernández y Díaz, 2001). 


\section{Resultados de la investigación}

El presente apartado expone los principales resultados de la investigación. El primero de ellos, los principales receptores y oferentes de la "iniciativa Ayuda al Comercio", liderada por la Organización Mundial del Comercio; el segundo resultado, corresponde al Índice de Competitividad Internacional propuesto a partir de la metodología empleada. Finalmente, la relación entre Ayuda al Comercio brindada a los PMA y la competitividad internacional, de estos países.

\section{Principales receptores y oferentes de la Ayuda para el Comercio}

La OMC y la Organización de Cooperación para el Desarrollo (OCDE) toma en cuenta la Inversión Extranjera Directa, remesas, otras ayudas oficiales y la Ayuda Oficial al Desarrollo (AOD) dentro de las entradas de financiamiento externo para medir la ayuda. Sin embargo, para efectos de esta investigación se toma en consideración los porcentajes específicos destinados para la Ayuda para el Comercio de los totales de Otras ayudas oficiales y de la Ayuda Oficial al Desarrollo. De manera tal, que al sumar dichos porcentajes entendidos como los entradas de financiamiento externo, las corrientes de ayuda de los donantes y los desembolsos de la ayuda por sector ${ }^{6}$ dentro del Total de la Ayuda para el Comercio de los países menos adelantados, miembros de la OMC (Figura 1), se puede observar en general un claro progreso en las corrientes de ayuda por donante de la mayoría de los treinta y seis PMA en estudio.

La Ayuda para el Comercio constituye la sumatoria de los porcentajes de otras ayudas oficiales y de la Ayuda Oficial al Desarrollo destinadas específicamente para mejorar el intercambio comercial de los PMA. De manera tal, que el monto destinado a la ayuda al comercio se constituye por los entradas de financiamiento externo, las corrientes de ayuda de los donantes y los desembolsos de la ayuda por sector (los cuales son: políticas y regulaciones comerciales, facilitación del comercio, transporte y almacenamiento; comunicaciones, generación y suministros de energía; negocios y otros servicios; servicios bancarios y financieros; industria, recursos minerales, turismo y ajustes relativos al comercio). La figura 1 , muestra el total de la ayuda para el comercio de los treinta y seis PMA, miembros de la OMC.

Los tres principales países que recibieron más Ayuda para el Comercio en el año 2013 son Bangladesh, con 2070 millones de USD, Tanzania con un importe cercano a los 1681 millones USD y República del Congo con 1528 millones USD. Si bien Angola para el año 2006 no registra flujos

${ }^{6}$ Los sectores contemplados en la categoría de Desembolsos de ayuda por sector son: políticas y regulaciones comerciales, facilitación del comercio, transporte y almacenamiento; comunicaciones, generación y suministros de energía; negocios y otros servicios; servicios bancarios y financieros; industria, recursos minerales, turismo y ajustes relativos al comercio.

12

Kendall Ariana López Peña y Raúl Gerardo Fonseca Hernández

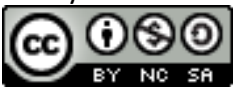

Revista Economía y Sociedad by Universidad Nacional is licensed under a CreativeCommons Reconocimiento-NoComercial- 
totales de ayuda para el comercio, para el año 2013 presenta un total de ayuda de 103 millones USD. Bangladesh por su parte registra el aumento más considerable de ayuda al pasar de 963 millones USD a 2070 millones USD y asimismo Mauritania de 18 millones USD a 198 millones. No obstante, Malí, Vanuatu y Yemen a pesar del compromiso asumido en Ginebra Suiza (diciembre, 2011) de mantener los niveles de Ayuda para el comercio 2006/08 y la cooperación con otros organismos reflejan una disminución de la ayuda entre los años 2006 y el año 2013 de 332 . 23 y 158 millones USD respectivamente para el último año. Y en el caso particular de Zambia no refleja datos de ayuda tanto para el año 2006 como el año 2013.

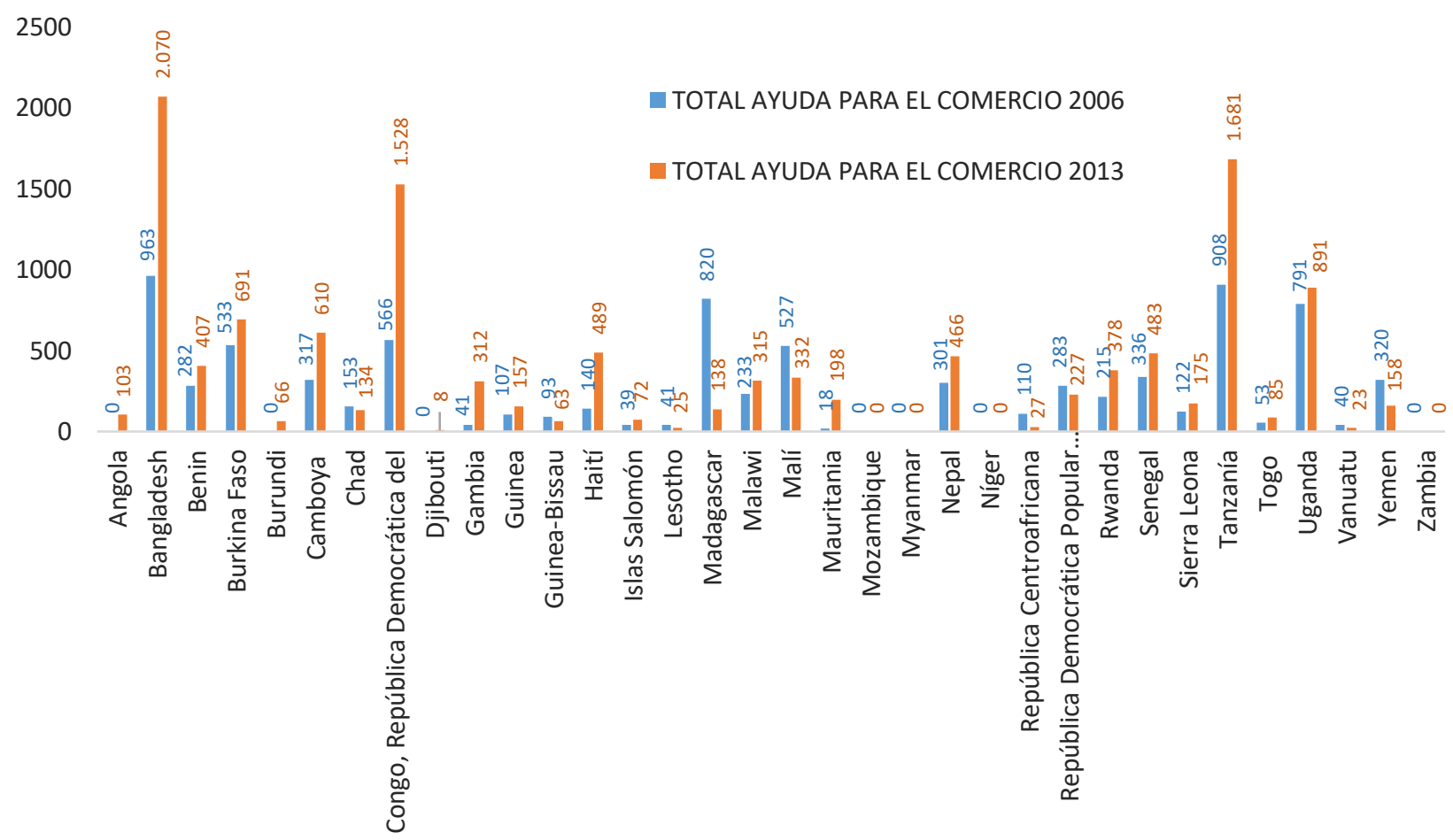

Figura 1. Total de ayuda al comercio de los PMA, según miembros de la OMC, 2006 - 2013. Cifras en millones de dólares. Fuente: Elaboración propia a partir de CIA (2014b) y OECD \& WTO (2015).

Por su parte, Benín, se suma a los países con progresos importantes en el total de la ayuda para el comercio y refleja un aumento de 125 millones USD para el 2013 en comparación con el año 2006, cuando se operativiza la Iniciativa de Ayuda al Comercio, resultado de la Conferencia de Hong Kong (2005). La Figura $\underline{2}$ muestra la ayuda recibida en calidad de Flujos corrientes de ayuda por Donante; en donde 162.8 millones USD del Total de 407 millones USD de Ayuda para el Comercio, corresponden a la ayuda brindada por los donantes. Siendo la Asociación Internacional de Fomento (IDA por sus siglas en inglés) con 77.4 millones de dólares para el año 2013; y el Banco de Desarrollo Africano (AfDF por sus siglas en inglés) con 35.3 millones USD los principales donantes. 


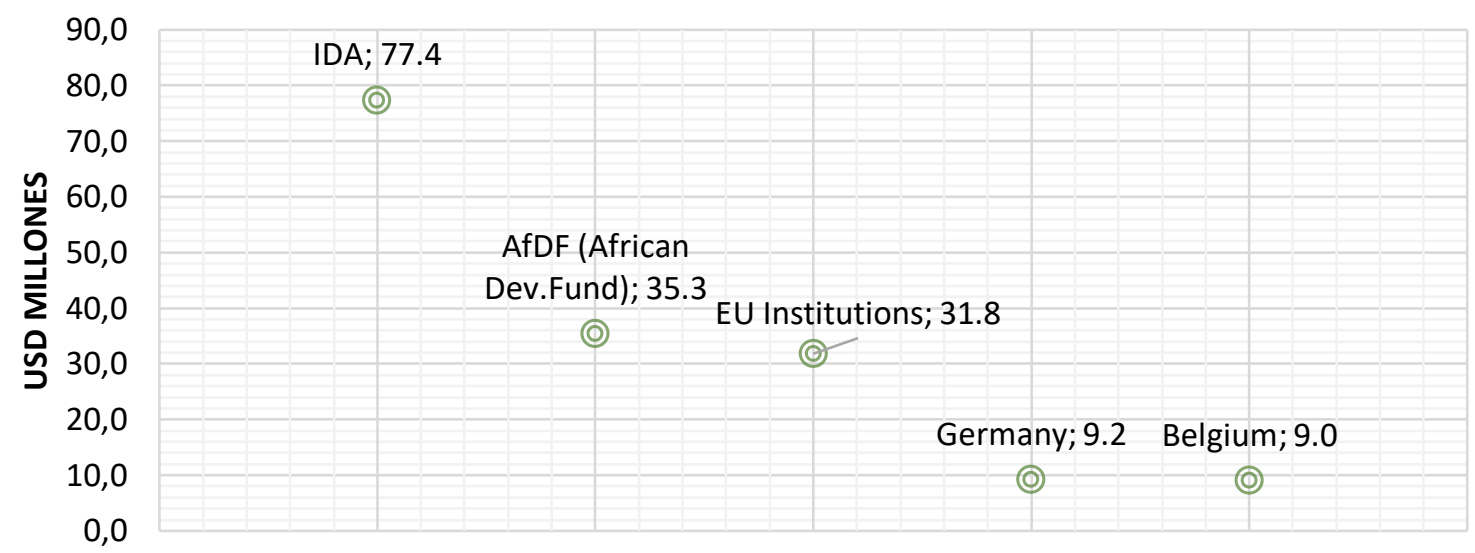

Figura 2. Benín. Flujos corrientes de Ayuda por Donante, (2013). Fuente: Elaboración propia; a partir de CIA (2014a) y OECD \& WTO, (2015).

Tomando en consideración, las cantidades (millones USD) y países receptores de la ayuda, se determina que la IDA y las instituciones de la Unión Europea son los principales donadores de la Ayuda para el Comercio en el año 2013.

La Tabla 2, muestra los principales donadores a la Ayuda para el Comercio, así como los países a los que aportaron en 2013.

\section{Tabla 2}

Principales donantes de la Ayuda para el Comercio, 2013

\begin{tabular}{|c|c|c|}
\hline Donador & Países receptores & $\begin{array}{l}\text { Flujos de Ayuda (USD } \\
\text { millones) }\end{array}$ \\
\hline $\operatorname{IDA}^{7}$ & $\begin{array}{l}\text { Benin, Bangladesh, Burkina Baso, Chad,Congo, Gambia, } \\
\text { Guinea, Guinea-Bissau, Lesotho, Madagascar, Malawi, Mali, } \\
\text { Mauritania, República Central Africana, Rwuanda, Sierra } \\
\text { Leona, Uganda, Vanuatu, Yemen. }\end{array}$ & 1827.3 \\
\hline $\begin{array}{l}\text { EU } \\
\text { Instituciones }\end{array}$ & $\begin{array}{l}\text { Chad, Congo, Gambia, Guinea, Guinea-Bissau, Islas Salomón, } \\
\text { Lesotho, Senegal, República Central Africana, República } \\
\text { Democrática Lao, Nepal, Uganda, Madagascar, Malawi, } \\
\text { Rwuanda, Togo, Tanzania. }\end{array}$ & 486.3 \\
\hline
\end{tabular}

Fuente: Elaboración propia según datos; a partir de OECD \& WTO (2015)

\footnotetext{
${ }^{7}$ Asociación Internacional de Fomento (IDA por sus siglas en inglés)
}

14

Kendall Ariana López Peña y Raúl Gerardo Fonseca Hernández

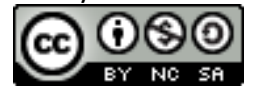

Revista Economía y Sociedad by Universidad Nacional is licensed under a CreativeCommons Reconocimiento-NoComercial- 
Finalmente, tomando en consideración los tres principales países que recibieron más Total de Ayuda para el Comercio en el año 2013; Bangladesh 2070 millones de USD, Tanzania 1681 millones USD y República del Congo con 1528 millones USD (Figura 1). Se observa que cada uno de los países antes mencionados presentan un progreso considerable en la categoría de los flujos corrientes por donante, en particular la República Democrática del Congo, al pasar de 182 millones USD en el año 2006 a 596 millones USD para el año 2013. Cuyos principales donantes además de IDA se destacan las instituciones de la UE y el AfDF; presentando Reino Unido un aumento importante en los flujos corrientes de ayuda que otorga para dicho país en el año 2013 (8.8 millones USD para el año 2006 y 33.6 millones USD para el año 2013). Pero disminuyendo en 8.8 millones USD la ayuda que brindaba a Tanzania para el año 2013.

\section{Índice de Competitividad Internacional}

El Índice de Competitividad Internacional propuesto en el presente documento, expone que en general todos los 34 países presentan entre una baja y poca competitividad. El resultado del índice para cada PMA se muestre en la Figura $\underline{3}$.

Se observa que ningún país muestra una alta competitividad. No obstante, en el continente africano solo 8 países de los 25 de la región poseen una competitividad media, los restantes 17 se caracterizan por tener una baja y poca competitividad. La competitividad media de la región africana está asociada a Mauritania, Chad, Lesotho, Ruanda, Madagascar, Guinea, Malawi y Uganda; con $0.60,0.59,0.57,0.56,0.55,0.52$ y 0.51 respectivamente. Esta se explica por un alto dinamismo en la competitividad interna y la apertura comercial en la mayoría de los países antes mencionados. Con la excepción de Chad, que se encuentra en la posición tres del ranking; en donde el principal dinamismo se presenta en la eficiencia de las exportaciones con un $26 \%$; es decir su alta competitividad se encuentra vinculada al costo y tiempo de las exportaciones que realiza. 


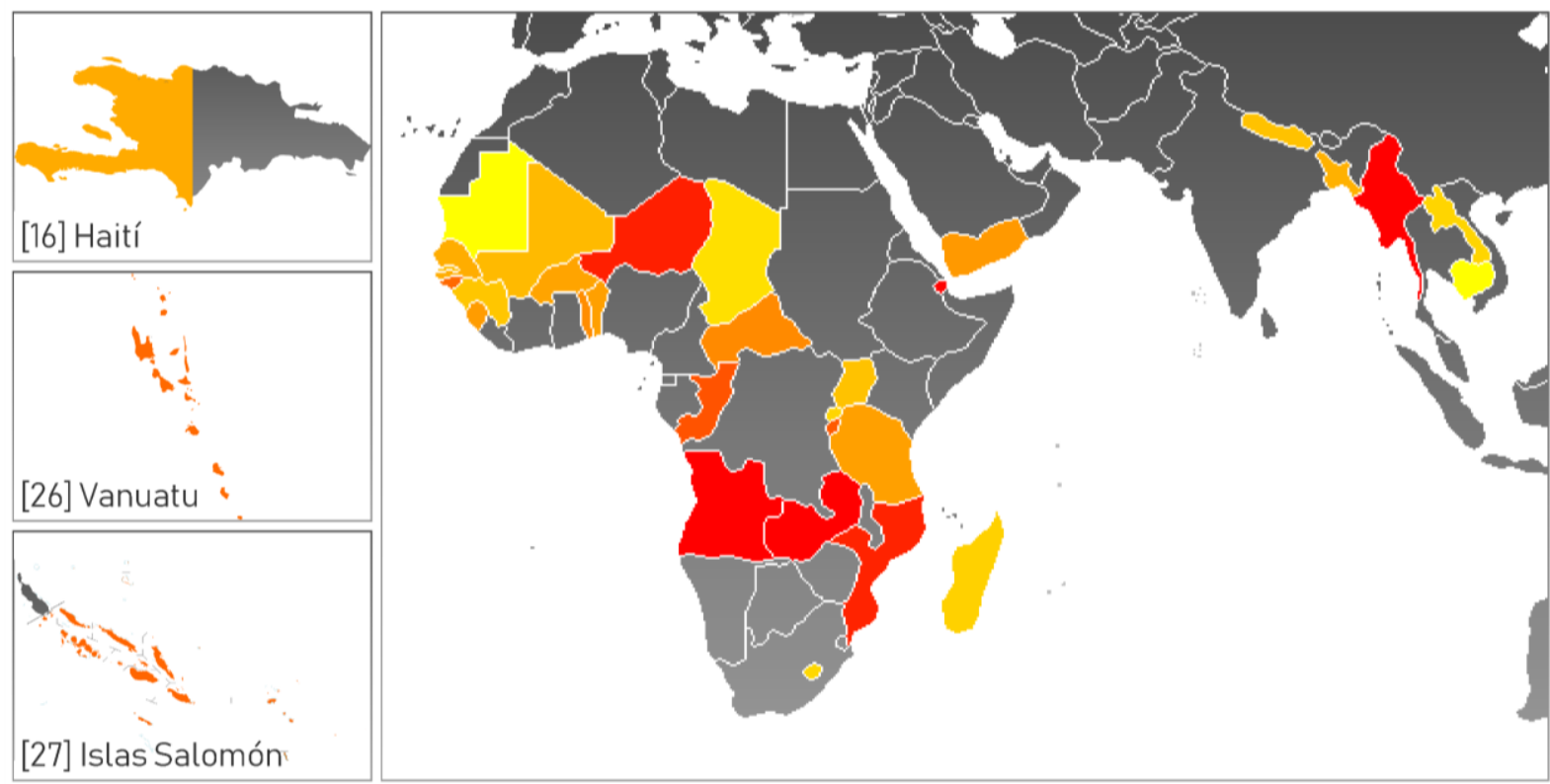

0.00

\subsection{0}

1: Escala de colores: El color amarillo representa la calificación más alta (igual o cercana al 0,60 ), mientras que el color rojo representa la calificación más baja (igual o cercana al 0,00).

2: Escala de medición: Competitividad media (entre 0,75-0,51), poca competitividad $(0,50-0,26)$ y baja competitividad $(0,25-0,00)$.

Figura 3. Ranking del Índice Competitividad Internacional de los PMA, miembros de la OMC. $2013^{8}$. Fuente: elaboración propia.

En general, los treinta y cuatro países presentan entre una baja y poca competitividad según la clasificación por quintiles, por tratarse de países menos adelantados; sin embargo, es importante destacar que entre los mismos PMA, algunos presentan un ICI más alto o más bajo que otros. Por lo tanto, según la escala de medición, específicamente para los países menos adelantados de la Figura $\underline{3}$, se observa que ningún país muestra una alta competitividad. No obstante, en el continente africano solo 8 países de los 25 de la región poseen una competitividad media, los restantes 17 se caracterizan por tener una baja y poca competitividad. La competitividad media de la región africana está asociada a Mauritania, Chad, Lesotho, Ruanda, Madagascar, Guinea, Malawi y Uganda; con $0.60,0.59,0.57,0.56,0.55,0.52$ y 0.51 respectivamente. Esta se explica por un alto dinamismo en la competitividad interna y la apertura comercial en la mayoría de los países antes mencionados. Con la excepción de Chad, que se encuentra en la posición tres del ranking; en donde el principal dinamismo se presenta en la Eficiencia de las exportaciones con un

\footnotetext{
${ }^{8}$ Ver apéndice 1.
}

16 
$26 \%$; es decir su alta competitividad se encuentra vinculada al costo y tiempo de las exportaciones que realiza.

Los puestos más bajos del ICl son ocupados por Angola y Zambia con 0.04 que los colocan en las últimas posiciones del ranking. Dichos países no presentaron datos en los indicadores de eficiencia de las exportaciones, competitividad interna e Índice de Desarrollo Humano y únicamente mostraron un $4 \%$ en el indicador de apertura comercial. Vanuatu por su parte, que presenta una baja competitividad con un 0.28 posee una deficiencia en la apertura comercial, resultado de un bajo PIB; así como de las exportaciones y un aumento de las importaciones. Asimismo, una limitada actividad en la eficiencia de las exportaciones (costo y tiempo de las exportaciones) y ningún registro de datos para la competitividad interna. Sin embargo, cabe destacar que a pesar de poseer una baja competitividad, Vanuatu se posiciona entre los PMA con mayores Índices de Desarrollo Humano con un $18 \%$.

El determinante de la competitividad internacional, según el $\mathrm{ICl}$ es la competitividad interna desarrollada en los países menos adelantados. No obstante, se resalta que tanto Mauritania y Camboya (posición 1 y 2 del ranking, respectivamente) presentan también altos Índices de Desarrollo Humano entre los PMA en estudio, junto a Vanuatu que posee una baja competitividad. Por lo tanto, la competitividad interna posee una mayor importancia en la competitividad internacional, en comparación con los resultados del Índice de Desarrollo Humano de cada uno de los países menos adelantados.

\section{Relación entre la Ayuda para el Comercio y la competitividad internacional}

Por otra parte, con respecto a la correlación entre el total de Ayuda para el Comercio en relación con el Índice de Competitividad Internacional de los países menos adelantados, se concluye que existe una correlación de 0.9 , es decir, no existe una relación lineal entre ambas variables. De tal manera, posee un rango de nula relación o también una relación no lineal. Por lo tanto, no existe una relación entre el total de la Ayuda para el Comercio otorgada por la OMC en términos de los flujos de financiamiento externo, la ayuda otorgada por los donantes y la ayuda por sectores con respecto al Índice de Competitividad Internacional (ICI) que posee cada uno de los treinta seis países menos adelantados, miembros de la organización.

En la Tabla $\underline{3}$ se puede observar dicha correlación de Pearson entre la Ayuda para el Comercio y el Índice de Competitividad Internacional de los 36 países menos adelantados, miembros de la OMC. 
Tabla 3

Correlación de Pearson entre la Ayuda para el Comercio y el Índice de Competitividad Internacional

\begin{tabular}{lc}
\hline Componentes de la competitividad internacional & Correlación \\
\hline 1. Correlación general & 0.09 \\
2. IDH & 0.11 \\
3. Competitividad interna & 0.10 \\
4. Eficiencia de las exportaciones & 0.04 \\
5. Apertura comercial & 0.01 \\
\hline
\end{tabular}

Fuente: elaboración propia

Se puede observar que, aunque existe una escasa relación en términos generales; al desagregar la correlación entre ambas variables (Ayuda para el Comercio y Competitividad Internacional), se puede apreciar que sí existe una relación un poco más fuerte entre la Ayuda para el Comercio y la competitividad interna, cuyo rango de relación es de 0.10 . Asimismo, una correlación aún más fuerte entre la Ayuda para el Comercio y el Índice de Desarrollo Humano (IDH), cuyo rango es de 0.11 .

Por lo tanto, se puede afirmar que la Ayuda para el Comercio en términos generales es deficiente, desde el punto de vista de aumentar la competitividad; sin embargo, al calcular específicamente la relación de la Ayuda otorgada en cada uno de los indicadores (subvariables) del índice de Competitividad Internacional, se logra observar un efecto más considerable en el Índice de Desarrollo Humano (IDH) de los PMA.

Por otra parte, al realizar el análisis de correlación de Pearson aplicado específicamente a los indicadores de la subvariable de competitividad interna (Tabla 4) se observa que, si se invierte mayoritariamente la Ayuda para el Comercio en acceso a préstamos, dirigidos a infraestructura portuaria, para garantizar suministros de electricidad y en carreteras e infraestructura aeroportuaria, se puede lograr una mayor efectividad en el Índice de Competitividad Internacional de los PMA en estudio.

Tabla 4

Análisis de correlación de Pearson en los indicadores de Competitividad interna

\begin{tabular}{lc}
\hline \multicolumn{1}{c}{ Área } & Rango \\
\hline Acceso a préstamos & 0.29 \\
Infraestructura portuaria & 0.20 \\
Suministros de electricidad & 0.14 \\
Carreteras & 0.12 \\
Infraestructura aeroportuaria & 0.12 \\
\hline
\end{tabular}

Fuente: Elaboración propia.

18

Kendall Ariana López Peña y Raúl Gerardo Fonseca Hernández

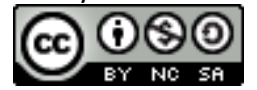

Revista Economía y Sociedad by Universidad Nacional is licensed under a CreativeCommons Reconocimiento-NoComercial- 
En síntesis, si los flujos totales de Ayuda para el Comercio se invierten más en los puntos con mayor correlación (acceso a préstamos y a infraestructura portuaria), aumentará más la Competitividad interna y por tanto el Desarrollo Humano. De esta forma la Ayuda para el Comercio otorgada por la OMC impactará en mayor medida el Índice de Desarrollo Humano de los países menos adelantados (PMA), miembros de la organización.

\section{Conclusiones y recomendaciones}

La teoría institucionalista ha intentado explicar cómo la cooperación perdura sin la legalización. Dicha definición contiene tres criterios: "el grado en que las reglas son obligatorias, la precisión de esas reglas y la delegación de algunas funciones de interpretación, monitoreo e implementación a un tercero". (Goldstein, Goldstein, et al., 2000, p. 387) Es así que, desde el concepto de Abbot, Keohane, et al., (citado por Goldstein, et al., 2000) afirman: "una institución plenamente legalizada es una instituación con altos niveles de obligación, precisión y delegación" (p. 396). En ese sentido, queda en evidencia que la OMC tanto régimen internacional en sí mismo, como régimen internacional de cooperación, específicamente en materia de Ayuda para el Comercio, presenta un alto grado de institucionalización y de manera particular, de legalización. Debido a los altos niveles de obligatoriedad y precisión que tienen las reglas derivadas de los Acuerdos y las funciones de monitoreo que emite para lograr la trasparencia entre sus miembros. Por tanto, generan expectativas de una cooperación a futuro para sus respectivos miembros; en especial para los países en desarrollo y los PMA.

Al analizar por separado aquellas subvariables determinantes de la competitividad internacional, se deduce que según el Índice de Competitividad Internacional (ICI) propuesto, es la competitividad interna la subvariable determinante y por esta razón, el IDH o índice de desarrollo humano no representa necesariamente que el PMA posea una media o alta competitividad. Sin embargo, al realizar la correlación por el método de Pearson se logra observar la existencia de una relación más fuerte entre la Ayuda para el Comercio y el Índice de Desarrollo Humano (IDH). Por consiguiente, si se analiza por separado la competitividad internacional de los PMA, el Índice de Desarrollo Humano no resulta determinante en la competitividad de dichos países. No obstante, según el Coeficiente de Pearson si se correlaciona con la ayuda, se podría deducir cierta relación entre la Ayuda para el Comercio y el Índice de Desarrollo Humano.

Desde otra perspectiva, al analizar la asociación entre las dos variables cuantitativas (Ayuda al Comercio y Competitividad Internacional) mediante el método de correlación de Pearson, para determinar la relación general entre ambas variables, se puede concluir, según la escala de medición, una escasa o nula relación entre el total de la Ayuda para el Comercio otorgada por la $\mathrm{OMC}$,en términos de las Entradas de financiamiento externo, la ayuda otorgada por los donantes y la ayuda por sectores, con respecto al Índice de Competitividad Internacional (ICI) que posee cada uno de los treinta seis países menos adelantados miembros de la organización. Es 
importante destacar, que tal y como se mencionó anteriormente, al correlacionar el total de la ayuda con las subvariables de la competitividad internacional, se observa una relación un poco más fuerte entre la Ayuda para el Comercio y la competitividad interna, cuyo rango de relación es de 0.10. Asimismo, una correlación aún más fuerte entre la Ayuda para el Comercio y el Índice de Desarrollo Humano (IDH), cuyo rango es de 0,11 .

De ese modo, pese al impacto relativo en la competitividad internacional, la efectividad de la iniciativa "ayuda al comercio" puede mejorar si los recursos se orientan, específicamente, a la competitividad interna y el IDH; de manera particular en infraestructura; esta es la variable que mejora en mayor medida la competitividad interna. Para ello debe invertirse en infraestructura portuaria, suministros de electricidad, carreteras e infraestructura aeroportuaria respectivamente.

\section{Referencias}

Banco Mundial. (s.f.). Observatorio de Competitividad. Recuperado de http://www.competitividad.org.do/posicionamiento/banco-mundial/

Banco Mundial. (2016). Alemania lidera el índice de desempeño logístico 2016. Recuperado de http://www.bancomundial.org/es/news/press-release/2016/06/28/germany-tops2016-logistics-performance-index

Barbé, E. (2007). Relaciones Internacionales. Tercera edición. Madrid: Tecnos.

Castellanos, C., Castellanos, J., Machado, N., Vila, Z. y Barbosa, G. (2012). Contribución de los clusters a la competitividad de las empresas. Revista Técnica Administrativa, 50(11), 1-30. Recuperado de http://www.cyta.com.ar/ta1102/v11n2a2.htm

Central Intelligence Agency (2014a). The World Factbook. Exports. Recuperado de https://www.cia.gov/library/publications/the-world-factbook/fields/2078.html\#bn

Central Intelligence Agency (2014b). The World Factbook. Imports. Recuperado de https://www.cia.gov/library/publications/the-world-factbook/fields/2087.html\#bn

Central Intelligence Agency (2014c). The World Factbook. Gross Domestic Product. Recuperado de $\quad$ https://www.cia.gov/library/publications/the-worldfactbook/fields/2195.html\#bn 
Conferencia de las Naciones Unidas para el Comercio y Desarrollo (2015) ¿Cuáles son los países menos adelantados? Recuperado de http://unctad.org/es/Paginas/PressRelease.aspx?OriginalVersionID=277

Fernández, P \& Díaz, P. (2001). Relación entre variables cuantitativas. Unidad de Unidad de Epidemiología Clínica y Bioestadística, (4), 141-144. Recuperado de https://www.fisterra.com/mbe/investiga/var cuantitativas/var cuantitativas2.pdf

Fonseca, R. (2012). Índice de Competitividad Territorial aplicado a los cantones de Heredia. Economía y Sociedad, 42(17), 75-98. Recuperado de http://www.revistas.una.ac.cr/index.php/economia/article/view/5119

Goldstein, J; Kahler, M; Keohane, R \& Slaghter, A. (2000). International Organization. Legalization and World Politics. 54(3): Introduction: Legalization and World Politics. 385-399.

IMD World Competitiveness Center (2014). IMD World Competitiveness Yearbook. Recuperado de http://www.colombiacompetitiva.gov.co/prensa/informes/IMD WCY-2014.pdf

Keohane, R., \& Nye, J. (1988). Poder e Interdependencia. La política mundial en transición. Buenos Aires, Argentina: Grupo Editor Latinoamericano. Recuperado de https://books.google.co.cr/books/about/Poder_e interdependencia.html?id=LAJ1A AAACAAJ\&redir esc=y

Krasner, S. (1983). Structural causes and regimes consequences: regimes as intervening variables. International Regimes: Spring, 1982, 2(36), 185-205. Recuperado de https://www.jstor.org/stable/2706520?seq=1\#page_scan tab contents

López, K. (2017). La OMC como régimen internacional de cooperación en materia de Ayuda al Comercio en los países menos adelantados: Caso Haití. (Tesis inédita de maestría). Universidad Nacional de Costa Rica, Heredia.

Organización para la Cooperación y el Desarrollo \& World Trade Organization (2015). Reducing trade coste for inclusive, sustainable growth. Recuperado de https://www.wto.org/english/res e/booksp_e/aid4trade15 e.pdf

Organización Mundial del Comercio. (s.f., a). ¿Qué es la OMC? Recuperado de https://www.wto.org/spanish/thewto s/whatis s/tif s/fact1 s.htm

Organización Mundial del Comercio. (s. f., b). Declaración Ministerial. Recuperado de https://www.wto.org/spanish/thewto_s/minist_s/min05_s/final text_s.htm 
Organización Mundial del Comercio (2007). Programa de trabajo de la OMC sobre la ayuda para el comercio. Recuperado

de https://www.wto.org/spanish/tratop_s/devel_s/a4t_s/global review07 s.htm

Organización Mundial del Comercio (2016). Programa de Trabajo sobre Ayuda al Comercio. Recuperado https://www.wto.org/spanish/tratop_s/devel_s/a4t_s/aid4trade_s.htm

Porter, M. (2007). La ventaja competitiva de las naciones. Harvard business review, 11(85), 69-95. Recuperado de https://dialnet.unirioja.es/servlet/articulo?codigo=2469800

World Economic Forum (s.f). Competitiveness Rankings. Recuperado de http://reports.weforum.org/global-competitiveness-report-20152016/competitiveness-rankings/

World Trade Organization (2006). Recommendations of the Task force on Aid for Trade. Recuperado de https://www.odi.org/publications/4053-aid-trade 
Apéndice 1

Índice de Competitividad Internacional de los Países Menos Adelantados

\begin{tabular}{|c|c|c|c|}
\hline Miembro & Calificación & Miembro & Calificaciór \\
\hline Mauritania & 0,60 & Benín & 0,42 \\
\hline Camboya & 0,60 & Tanzania & 0,42 \\
\hline Chad & 0,59 & Sierra Leona & 0,41 \\
\hline Lesotho & 0,57 & Yemén & 0,40 \\
\hline Ruanda & 0,56 & Togo & 0,37 \\
\hline República Popular de Laos & 0,56 & República del Congo & 0,37 \\
\hline Madagascar & 0,55 & Republica Centroafricana & 0,36 \\
\hline Guinea & 0,52 & Guinea-Bissau & 0,28 \\
\hline Malawi & 0,51 & Vanuatu & 0,28 \\
\hline Nepal & 0,51 & Islas Salomón & 0,27 \\
\hline Uganda & 0,51 & Burundí & 0,24 \\
\hline Mali & 0,49 & Mozanbique & 0,13 \\
\hline Gambia & 0,49 & Niger & 0,12 \\
\hline Bangladesh & 0,46 & Djibouti & 0,07 \\
\hline Senegal & 0,45 & Angola & 0,04 \\
\hline Haití & 0,44 & Zambia & 0,04 \\
\hline Burkina Faso & 0,43 & Birmania & 0,04 \\
\hline
\end{tabular}

Nota: Escala de medición: Competitividad media (entre 0,75-0,50), poca competitividad $(0,50-0,26)$, y Baja competitividad $(0,25-0)$.

Fuente: Elaboración propia 